\title{
Sentinel Node Navigation Surgery in Upper Gastrointestinal Cancer: What Can It Teach Us?
}

\author{
Hiroya Takeuchi, MD and Yuko Kitagawa, MD \\ Department of Surgery, Keio University School of Medicine, Tokyo, Japan
}

The histopathological status of regional lymph nodes is one of the most significant predictors of recurrence and overall survival for most solid tumors and often is used to justify stratification of patients for adjuvant therapy. More accurate staging, efficient diagnosis of lymphatic spread, and prognostic information can be obtained from a small number of lymph nodes, by intraoperative sentinel node (SN) mapping and sentinel lymphadenectomy. The sentinel node navigation surgery (SNNS) is defined as a novel minimally invasive surgery that can avoid unnecessary lymph node dissection based on SN biopsy and diagnosis for nodal metastasis targeting $\mathrm{SN}$.

SN mapping and biopsy was first applied to melanoma and breast cancer and was subsequently extended to many other solid tumors. ${ }^{1-6}$ The clinical applications of SN mapping for upper gastrointestinal (GI) cancer have been controversial. However, the past 10 years have witnessed the recognition of several studies supporting the validity of the SN concept for early-stage upper GI cancer. Single, institutional results of SN mapping for early-stage esophageal cancer or gastric cancer are being increasingly considered acceptable in terms of detection rate and accuracy to predict regional lymph node status. ${ }^{7-9}$

The current article by Uenosono et al. ${ }^{10}$ reports on SN mapping in esophageal cancer. The authors evaluated the feasibility and accuracy of radioguided SN mapping of the 134 patients with clinical T1 to T3 esophageal cancer. They concluded that radioguided SN mapping is an accurate diagnostic procedure for detecting lymph node metastasis in patients with clinical T1N0 esophageal cancer. Also Märkle et al. ${ }^{11}$ have indicated that ex vivo SN mapping would provide reliable staging information based on more focused

(C) Society of Surgical Oncology 2011

Published Online: 19 April 2011

H. Takeuchi, MD

e-mail: htakeuch@sc.itc.keio.ac.jp histopathology of SN in patients with gastric cancer. These results suggest that $\mathrm{SN}$ mapping may be a promising diagnostic tool for proper staging in early-stage upper GI cancer. Accurate and sensitive detection of micrometastasis assessed by SN mapping is very important clinically in aiding the performance of adequate adjuvant chemotherapy for $\mathrm{cN} 0$ esophageal cancer or gastric cancer.

In addition, SNNS might herald a new era for upper GI cancer. Minimized esophagectomy or gastrectomy with individualized selective and modified lymphadenectomy for early upper GI cancer with negative SN is expected to become feasible and clinically useful as less invasive surgical procedures. However, the clinical applications of SNNS seem to be slightly different between esophageal cancer and gastric cancer because of the distribution of SN. Uenosono et al. ${ }^{10}$ revealed that $\mathrm{SN}$ in thoracic esophageal cancer was distributed from the cervical to the abdominal areas, especially in upper and middle thoracic esophageal cancers. Their findings suggested that the clinical application of SN mapping in esophageal cancer should not be limited to less invasive surgery. ${ }^{9}$ For instance, if $\mathrm{SN}$ are pathologically positive for metastasis, lymphadenectomy of the SN basin should be performed carefully and intensively. In particular, if SN are identified along the recurrent laryngeal nerves in the upper mediastinum and are positive for metastasis, extended lymphadenectomy for the upper mediastinum and/or additional cervical lymphadenectomy as a three-field lymphadenectomy may be considered. On the other hand, if SN were pathologically negative, unnecessary cervical lymphadenectomy could be omitted. Also, if the SN were identified only in the abdominal area and pathologically negative in the case with adenocarcinoma of distal esophagus, the patient would be treated with limited resection of distal esophagus by transhiatal approach without extensive mediastinal lymph node dissection. ${ }^{12}$

The anatomical situation of the stomach is relatively suitable for SN mapping compared with that of esophageal 
cancer. The distribution of $\mathrm{SN}$ and sentinel lymphatic basins is known to be relatively limited to the periphery of the stomach. Therefore, SNNS would be useful in deciding on the extent of gastric resection. Appropriate indications for function-preserving surgery, such as partial resection, segmental gastrectomy, pylorus-preserving gastrectomy, and proximal gastrectomy for cNO early-stage gastric cancer, could be individually determined based on SN status. ${ }^{13}$ Various types of laparoscopic function-preserving surgery are applicable for cases with cancer-negative SN. ${ }^{14}$ Earlier recovery after surgery and preservation of quality of life in the late phase can be achieved by laparoscopiclimited gastrectomy with SN navigation. A combination of endoscopic treatments, such as endoscopic mucosal resection (EMR) and endoscopic submucosal dissection (ESD) and laparoscopic SN biopsy for superficial gastric cancer, is another attractive option as a novel minimally invasive approach. ${ }^{15}$ However, it is likely too early for laparoscopic SN mapping and consequent intraoperative EMR/ESD for SN-negative cases to become an accepted approach in clinical practice at this moment.

Several important issues such as the standardization of SN mapping procedures and the accuracy of intraoperative histopathology (or molecular diagnosis) to be resolved for future clinical applications of SNNS remain. Furthermore, well-designed feasibility studies of SN mapping for earlystage upper GI cancer by multicenter, prospective trials should be considered as the next step, because SN mapping has to be extremely accurate as a diagnostic tool for identification of clinically undetectable lymph node metastasis. Nonetheless, we desire that SN mapping will have proven to be a promising strategy for less invasive individualized management in patients with upper GI cancer.

\section{REFERENCES}

1. Morton DL, Wen DR, Wong JH, et al. Technical details of intraoperative lymphatic mapping for early stage melanoma. Arch Surg. 1992;127:392-9.
2. Giuliano AE, Kirgan DM, Guenther JM, Morton DL. Lymphatic mapping and sentinel lymphadenectomy for breast cancer. Ann Surg. 1994;220:391-401.

3. Yoshino I, Nakanishi R, Osaki T, et al. Unfavorable prognosis of patients with stage II non-small cell lung cancer associated with macroscopic nodal metastases. Chest. 1999;116:144-9.

4. Morton DL, Thompson JF, Essner R, Elashoff R, Stern SL, Nieweg OE. Validation of the accuracy of intraoperative lymphatic mapping and sentinel lymphadenectomy for early-stage melanoma: a multicenter trial. Multicenter Selective Lymphadenectomy Trial Group. Ann Surg. 1999;230:453-63.

5. Krag D, Weaver D, Ashikaga T, et al. The sentinel node in breast cancer-a multicenter validation study. $N$ Engl $J$ Med. 1998;339:941-6.

6. Bilchik AJ, Saha S, Wiese D, et al. Molecular staging of early colon cancer on the basis of sentinel node analysis: a multicenter phase II trial. J Clin Oncol. 2001;19:1128-36.

7. Kitagawa Y, Fujii H, Mukai M, et al. The role of the sentinel lymph node in gastrointestinal cancer. Surg Clin $N \mathrm{Am}$. 2000;80:1799-809.

8. Kitagawa Y, Fujii H, Mukai M, et al. Radio-guided sentinel node detection for gastric cancer. Br J Surg. 2002;89:604-8.

9. Takeuchi H, Fujii H, Ando N, et al. validation study of radioguided sentinel lymph node navigation in esophageal cancer. Ann Surg. 2009;249:757-63.

10. Uenosono Y, Arigami T, Yanagita S, et al. Sentinel node navigation surgery is acceptable for clinical T1 and N0 esophageal cancer. Ann Surg Oncol. 2011 (in press).

11. Märkle B, Moldovan A, Jähnig H, et al. Combination of ex vivo sentinel lymph node mapping and methylene blue-assisted lymph node dissection in gastric cancer: a prospective and randomized study. Ann Surg Oncol. 2011 (in press).

12. Omloo JM, Lagarde SM, Hulscher JB, et al. Extended transthoracic resection compared with limited transhiatal resection for adenocarcinoma of the mid/distal esophagus. Five-year survival of a randomized clinical trial. Ann Surg. 2007;246:992-1001.

13. Kitagawa Y, Saikawa Y, Takeuchi H, et al. Sentinel node navigation in early stage gastric cancer: updated data and current status. Scand J Surg. 2006;95:256-9.

14. Saikawa Y, Otani Y, Kitagawa Y, et al. Interim results of sentinel node biopsy during laparoscopic gastrectomy: possible role in function-preserving surgery for early cancer. World J Surg. 2006; 30:1962-8.

15. Takeuchi H, Saikawa Y, Kitagawa Y. Laparoscopic sentinel node navigation surgery for early gastric cancer. Asian J Endosc Surg 2009;2:13-7. 